\title{
ESMO 2019-personal highlights in gastrointestinal cancer
}

\author{
A. Amann
}

Received: 3 February 2020 / Accepted: 25 March 2020 / Published online: 22 April 2020

(C) The Author(s) 2020

\begin{abstract}
Summary This article summarizes important highlights regarding gastrointestinal cancer at the 2019 ESMO annual meeting. The choice of the abstracts was based on a personal view with a focus on different tumor entities of the gastrointestinal tract. Precision-medicine-guided therapy for cancer entities was one of the main topics at this year's ESMO. Furthermore, circulating tumor DNA (ctDNA) by performing liquid biopsy was again another hot topic, especially in guiding adjuvant chemotherapy in stage III colorectal cancer (CRC) patients. Finally, hope was stirred up again for immunotherapy in the indication of firstline treatment in nonresectable hepatocellular cancer (HCC). In summary, the astonishing progress in new drugs that has been made in recent years is still ongoing and will lead to better treatment for cancer patients.
\end{abstract}

Keywords Molecular phenotyping - Targeted therapy · ESMO meeting 2019 - Gastrointestinal cancer - Precision medicine

\section{Introduction}

This year's ESMO 2019 annual meeting took place in Barcelona from 27 September to 1 October 2019 under the slogan "Translating science into better cancer patient care". Taking this into account, the abstracts described below were chosen. Game-changing novel strategies were presented especially in cancer entities with sparse therapeutic options including BRAF

\footnotetext{
A. Amann ( $\bowtie)$

Department of Hematology and Oncology, Internal

Medicine V, Medical University of Innsbruck,

Anichstraße 35, 6020 Innsbruck, Austria

arno.amann@tirol-kliniken.at
}

V600E mutated colorectal cancer (CRC), cholangiocellular cancer and hepatocellular cancer (HCC).

These abstracts all include cohorts of cancer entities that were subdefined because of intense translational research in recent years with varying impact on therapeutic decisions. However, most of these treatments will only be an option for a small cohort of cancer patients.

\section{Advanced stage cholangiocellular carcinoma}

FIGHT-202: A. Vogel presented the first analyses of this phase II trial at the ESMO meeting [1]. In summary, this trial included patients with locally advanced or metastatic cholangiocellular carcinoma that progressed after $\geq 1$ prior treatment. These patients were assigned depending on their documented fibroblast growth factor (FGF) / fibroblast growth factor receptor (FGFR) gene status to three different cohorts: A: FGFR2 gene rearrangements/fusions, B: other FGF/ FGFR gene alterations, or C: no FGF/FGFR gene alterations. They received oral pemigatinib $13.5 \mathrm{mg}$ QD (21-day cycle; 2 weeks on, 1 week off) until disease progression or unacceptable toxicity. Primary endpoint was centrally confirmed objective response rate (ORR) in cohort A. Secondary endpoints consisted of ORR (cohorts $\mathrm{B}, \mathrm{A}+\mathrm{B}$, and $\mathrm{C}$ ), duration of response (DOR), disease control rate (DCR), progression-free survival (PFS), overall survival (OS), and safety. In the end, 146 patients were included (cohort A, $n=107$; B, $n=20$; C, $n=18,1$ undetermined). The patient cohorts displayed a median range of age of 59 (26-78) years and $61 \% / 39 \%$ had 1 and $\geq 2$ prior therapies, respectively.

ORR in cohort A was $35.5 \%$ (95\% CI $26.5-45.4 \%$ ) with 3 complete responses and median (m)DOR was 7.5 months (95\% CI 5.7-14.5). DCR accounted for $82 \%$ (95\% CI 74-89\%) with a mPFS and mOS of 6.9 months 
(95\% CI 6.2-9.6) and 21.1 months (14.8-not reached) (OS not mature at cutoff), respectively. Notably, no patient achieved a response in cohorts B and C.

Most common adverse events were low grade including hyperphosphatemia $(60 \%$; grade $\geq 3,0 \%)$, alopecia $(49 \% ; 0 \%)$, diarrhea $(47 \% ; 3 \%)$, fatigue $(42 \%$; $5 \%$ ), nail toxicities (42\%; $2 \%$ ), and dysgeusia $(40 \%$; $0 \%)$.

In conclusion, this trial showed promising results for pemigatinib with an ORR of $35 \%$ in patients where usually no standard treatment option is available after progression in first-line treatment. However, pemigatinib only showed efficacy in patients with FGFR2 gene rearrangements/fusions. This alteration accounts for up to only $13 \%$ of all patients with cholangiocellular carcinoma. Therefore, pemigatinib will be only a treatment option in a small cohort of patients.

\section{Advanced stage hepatocellular carcinoma}

G030140 STUDY: At the meeting, M. Lee presented an update of the GO30140 trial [2]. The first results were presented at the ESMO Asia 2018 meeting one year ago [3]. In general, this phase $1 \mathrm{~b}$ trial included patients with an Eastern Cooperative Oncology Group (ECOG) Performance Status (ECOG-PS) of 0 tol with unresectable hepatocellular carcinoma (HCC) that had not received any previous treatment. Patient were randomized into two different arms (Arm A and F). In Arm F, patients were randomized 1:1 to atezolizumab (atezo) + bevacizumab (bev; F1) or atezo monotherapy (F2) and received atezo $1200 \mathrm{mg}$ IV q3w (21-day cycle; application day one), bev $15 \mathrm{mg} / \mathrm{kg}$ IV q3w until unacceptable toxicity or loss of clinical benefit. In Arm A, patients only received atezo + bev without a randomized control arm. Primary endpoints consisted of PFS (Arm F) and ORR (Arm A) by independent review facility (IRF)-assessed RECIST 1.1 and safety (Arm F and A)

This year's late-breaking abstract included the first randomized dataset evaluating atezo (anti-PD-L1) as monotherapy vs the combination of atezo + bev (Arm F) and the updated ORR data of the single-arm atezo + bev (Arm A).

In all, 60 and 59 patients were randomized to Arm F1 and F2, respectively. Notably, Arm F showed a statistically significant improvement in mPFS for atezo + bev vs atezo (5.6 vs 3.4 months, HR $0.55,80 \%$ CI, $0.40-0.74, P=0.0108)$. Treatment-related adverse events (TRAE) occurred in $41(68 \%) \mathrm{F} 1$ patients and 24 (41\%) F2 patients, including grade 3-4 TRAEs in 12 (20\%) F1 and 3 (5\%) F2 patients, respectively.

The updated results for the 104 patients in Arm A showed an ORR of $36 \%$ (37 patients) with $76 \%$ ongoing.

The authors concluded that the combination of atezo + bev is a promising first-line treatment option for patients with unresectable HCC by meeting the primary endpoint (PFS) for atezo + bev vs atezo alone and because of the updated durable responses of Arm A. So far, ORRs of $36 \%$ has never been reported for a treatment option in patients with HCC. Nevertheless, we will have to wait for confirming data in the phase III IMbrave150 trial.

\section{Precision-guided adjuvant chemotherapy in colorectal cancer}

LBA30_PR: J. Taieb presented an analysis of circulating tumor DNA (ctDNA) from patients enrolled in the IDEA-FRANCE phase III trial $[4,5]$. In this phase III trial, colorectal cancer patients with stage III disease were randomized either to 3-month $\mathrm{m}$ (modified)FOLFOX6 (fluorouracil (5-FU), oxaliplatin, calciumfolinat)/CAPOX (capecitabine, oxaliplatin) or 6-month mFOLFOX6/CAPOX with the primary endpoint of disease-free survival (DFS). Liquid biopsy was performed on $805 / 2010$ patients in this trial. ctDNA was tested by using the detection of two methylated markers (WIF1 and NPY) by digital droplet PCR, accordingly. In the end, 109 (13.5\%) patients had proven circulating tumor DNA (ctDNA) in their blood. For this patient cohort, 2-year DFS was 64\%, compared to $82 \%$ in those who were ctDNA negative. In multivariate analysis including age, gender, microsatellite status (MSI), perforation, $\mathrm{T}$ stage, $\mathrm{N}$ stage and treatment arm, ctDNA was confirmed as an independent prognostic marker (adjusted HR 1.85; 95\%CI 1.31 to $2.61 ; p<0.001)$.

Adjuvant treatment for 6 months was superior to 3 months in both ctDNA- (HR 0.69; $95 \%$ CI 0.52 to 0.93 ; $p=0.015$ ) and ctDNA+ patients (HR 0.50; 95\%CI 0.27 to $0.95 ; p=0.033$ ). Notably, ctDNA+ patients treated for 6 months had a similar prognosis to ctDNA patients treated for 3 months.

The authors concluded that ctDNA assessment was confirmed to be an independent prognostic marker. However, analysis of ctDNA status comparing high/low-risk stage III colorectal cancer is still missing. Therefore, further data are still needed to suggest liquid biopsy-guided adjuvant treatment in stage III colorectal cancer.

\section{Treatment option for BRAF mutated metastatic colorectal cancer}

BEACON trial: J. Tabernero presented expanded results of the BEACON trial at the ESMO this year [6]. The first results of this randomized, 3-arm, phase 3 study were already shown earlier this year at the World Congress on Gastrointestinal Cancer 2019 [7]. The BEACON trial evaluated the combination of encorafenib (ENCO), binimetinib (BINI) and cetuximab (CETUX), either as triplet or doublet $(\mathrm{ENCO}+\mathrm{CETUX})$ vs. investigator's choice of FOLFIRI + CETUX in patients with BRAF V600E mutant metastatic colorectal cancer whose disease pro- 
gressed after $\geq 1$ prior treatment. At the ESMO, the efficacy comparison OS, ORR, depth of response, and PFS between the triplet vs doublet was shown. A total of 224 and 220 patients were randomized to the triplet or doublet regimen, respectively. Median OS for triplet and doublet was 9.0 (95\%CI: 8.0, 11.4) and 8.4 months (95\%CI: 7.5, 11.0) (HR: 0.79 [95\% CI: 0.59, 1.06]). ORR was up to $26 \%$ (95\%CI: $18 \%$, 35\%) for triplet and 20\% (95\%CI: 13\%, 29\%) for doublet. For patients with only one prior regimen, ORR was $34 \%$ (95\%CI: 23, 47) and 22\% (95\%CI: 14, 33) for triplet and doublet, respectively. Grade 3+ TRAEs were only slightly higher in the triplet arm with $58 \%$ vs $50 \%$. Rates of discontinuation due to a TRAE were also similar with $7 \%$ and $8 \%$ either in the triplet or doublet arm.

In conclusion, the authors stated that both the triplet and also the doublet combinations showed high activity in BRAF V600E mutated CRC patients. Furthermore, the triplet combination offered improved efficacy over the doublet combination with only a slight addition in manageable toxicities. However, OS and ORR results were not significant comparing the doublet to the triplet regimen. Furthermore, offering the doublet treatment instead of the triplet could help to reduce costs and the number of pills that patients have to take. Nevertheless, this analysis showed further convincing results for this drug combination in patients with CRC that is associated usually with poor prognosis.

\section{Take home message}

These abstracts highlight the need of precise profiling of our cancer patients, exemplarily shown here by testing for BRAF mutations, ctDNA or FGFR2 alterations to provide state of the art treatment.

Funding Open access funding provided by University of Innsbruck and Medical University of Innsbruck.

Conflict of interest A. Amann declares that he has no affiliations with or involvement in any organization or entity with any financial interest (such as honoraria; educational grants; participation in speakers' bureaus; membership, employment, consultancies, stock ownership, or other equity interest; and expert testimony or patent-licensing arrangements), or nonfinancial interest (such as personal or professional relationships, affiliations, knowledge or beliefs) in the subject matter or materials discussed in this manuscript.

Open Access This article is licensed under a Creative Commons Attribution 4.0 International License, which permits use, sharing, adaptation, distribution and reproduction in any medium or format, as long as you give appropriate credit to the original author(s) and the source, provide a link to the Creative Commons licence, and indicate if changes were made. The images or other third party material in this article are included in the article's Creative Commons licence, unless indicated otherwise in a credit line to the material. If material is not included in the article's Creative Commons licence and your intended use is not permitted by statutory regulation or exceeds the permitted use, you will need to obtain permission directly from the copyright holder. To view a copy of this licence, visit http://creativecommons.org/licenses/by/4.0/.

\section{References}

1. Vogel A, et al. FIGHT-202: A phase II study of pemigatinib in patients (pts) with previously treated locally advanced or metastatic cholangiocarcinoma (CCA). Ann Oncol. 2019;30:v876.

2. Lee M, et al. Randomised efficacy and safety results for atezolizumab (Atezo) + bevacizumab (Bev) in patients (pts) with previouslyuntreated, unresectablehepatocellular carcinoma (HCC). Ann Oncol. 2019;30:v875.

3. Pishvaian MJ, et al. Updated safety and clinical activity results from a phase Ib study of atezolizumab + bevacizumab in hepatocellular carcinoma (HCC). J Clin Oncol. 2018;36(15_suppl):4074-4074. https://doi.org/10. 1200/JCO.2018.36.15_suppl.4074

4. André T, et al. Three versus 6 months of oxaliplatin-based adjuvant chemotherapy for patients with stage III colon cancer: disease-free survival results from a randomized, open-label, international duration evaluation of Adjuvant (IDEA) France, phase III trial. JClin Oncol. 2018;36:1469-77.

5. Taieb J, et al. Analysis of circulating tumour DNA (ctDNA) from patients enrolled in the IDEA-FRANCE phase III trial: Prognostic and predictive value for adjuvant treatment duration. Ann Oncol. 2019;30:v867.

6. Tabernero J, et al. Encorafenib plus cetuximab with or without binimetinib for BRAF V600E-mutant metastatic colorectal cancer: Expanded results from a randomized, 3-arm, phase III study vs the choice of either irinotecan or FOLFIRI plus cetuximab (BEACON CRC). Ann Oncol. 2019;30:v868.

7. Kopetz S, et al. BEACON CRC: a randomized, 3-Arm, phase 3 study of encorafenib and cetuximab with or without binimetinib vs. choice of either irinotecan or FOLFIRI plus cetuximab in BRAF V600E-mutant metastatic colorectal cancer. Ann Oncol. 2019;30:iv154.

Publisher's Note Springer Nature remains neutral with regard to jurisdictional claims in published maps and institutional affiliations.

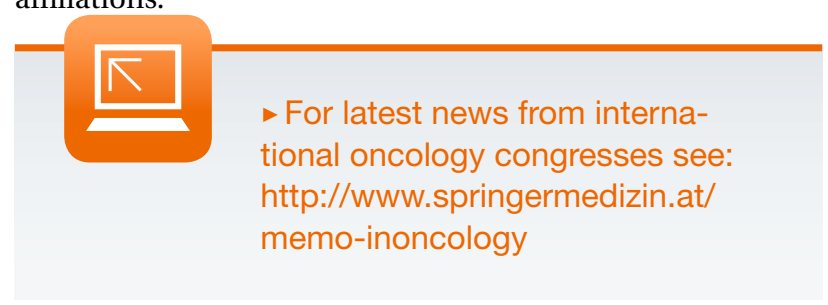

